\title{
ADJUSTED RECOMBINATION OPERATOR FOR SIMULATION-BASED CONSTRUCTION SCHEDULE OPTIMIZATION
}

\author{
Kamil Szczesny \\ Matthias Hamm \\ Markus König \\ Ruhr-Universität Bochum \\ Universitätsstraße 150 \\ Bochum, 44780, GERMANY
}

\begin{abstract}
An efficient execution of complex construction projects requires a comprehensive scheduling of all construction activities. For this, it is necessary to consider various restrictions and the availability of required resources. The generation of efficient schedules is a very challenging task, in fact an NP-hard optimization problem. An appropriate approach is the application of discrete-event simulation for the generation of valid schedules. Additionally, simulation is combined with optimization methods to determine efficient schedules regarding given objectives. The applied optimization techniques are evolutionary algorithms. Thus, operators have to be implemented that define the way of generating new schedules in the recombination step of the algorithm. In this paper an improved operator is presented that outperforms common operators for scheduling problems by considering technological dependencies between activities, so that it will be possible to determine efficient schedules for complex construction scheduling problems. An example of implementation is presented to validate the developed operator.
\end{abstract}

\section{INTRODUCTION}

The efficient execution of construction activities is absolutely essential for today's construction industry. A multitude of construction tasks has to be scheduled efficiently regarding different conflicting objectives like time, cost and quality. However, various constraints like resource restrictions or precedence relations have to be taken into consideration. For that reason, scheduling problems belong to the class of combinatorial optimization problems and the generation of efficient schedules for complex construction projects results in an NP-hard optimization problem. Thus, an analytical calculation of optimal schedules with exact mathematical methods is impractical.

In the paper at hand, an approach is presented where discrete-event simulation is used for the generation of valid schedules for construction projects. By implementing the constraint satisfaction approach within a discrete-event simulation framework, it is possible to generate schedules for highly complex projects under the consideration of all existing constraints in a relatively short amount of time. To enable the determination of efficient schedules, an existing optimization framework is adapted for the use in combinatorial optimization. The implemented optimization strategy is an evolutionary algorithm. Thus, several steps of the algorithm have to be defined, e.g. the recombination of selected solutions. We present a rankbased crossover operator, which has advantages over existing operators concerning the properties of generated solutions. In the paper at hand, this rank-based crossover operator is described in detail, the optimization framework is presented, and the efficiency of the developed operator is shown by a comprehensive case study. 


\section{Szczesny, Hamm, and König}

An overview of related research is given in section two, while the subsequent section contains a detailed description of the problem covered in this paper. In section four, the methodology of the developed operator is presented while in section five important implementation details are explained. A case study is presented in section six, where some results are shown and the adjusted operator can be validated. The last section gives a conclusion as well as an outlook regarding required future work.

\section{RELATED WORK}

As already mentioned, the generation of efficient schedules for complex construction projects is a very difficult task and the calculation of an optimal schedule is not possible. A common alternative to an analytical calculation is the application of heuristics which enables the determination of near-optimal schedules. Heuristics can solve a very general class of computational problems in an acceptable amount of time. Commonly applied heuristics are Simulated Annealing (Kirkpatrick et al., 1983), Evolutionary Algorithms (Bäck 1996) and Particle Swarm Optimization (Kennedy, Eberhart, and Shi 2001). For several years, the optimization of scheduling problems has been a research topic and various heuristics have been applied to solve these problems. Detailed literature reviews which give appropriate overviews of previous research about optimization methods can be found in Brucker et al. (1999), Hartmann and Kolisch (2000), Hartmann and Kolisch (2006) and Warren Liao et al. (2011). Several researchers have applied evolutionary algorithms to investigate differentiated aspects for scheduling problems. In Leu and Yang (1999) the authors proposed a crossover operator to tackle resource allocation for genetic algorithms. Chen and Weng (2009) included a time-cost trade-off mechanism to the resource allocation consideration. Senouci and Eldin (2004) applied genetic algorithms to solve scheduling problems with respect to resource leveling. Hybrid optimization concepts have been investigated, where evolutionary algorithms were coupled with other heuristics. Jaśkowski and Sobotka (2006) present a solving mechanism to overcome drawbacks that let existing scheduling methods fail to solve complex practical problems effectively. In their approach, Jaśkowski and Sobotka propose the integration of two heuristic methods, of which one considers resource allocation, while another genetic algorithm searches for the minimal project duration.

Heuristics are often combined with simulation concepts to generate a multitude of solutions for different combinations of parameters. The discrete-event simulation approach applied in this paper is based on the concept of Beißert, König, and Bargstädt (2007), where the Constraint Satisfaction Approach in combination with discrete-event simulation guarantees the observation of valid construction schedules. The application of simulation-based optimization is a possible technique to solve a scheduling problem as described in Hamm et al. (2011). The approach presented in this paper deals with three components: the optimization method component, the scheduling problem component and the discrete-event simulation component (see Figure 1). The discrete-event simulation component represents a simulation engine that is powerful enough for the computation of valid construction schedules for a given input. The scheduling problem component acts as a transformation layer between the optimization method and the discreteevent simulation, e.g. it transforms the input and output data of each component. The last component, the optimization method component, represents any optimization method that is suitable for solving scheduling problems. The optimization framework which is used in this paper is called MOPACK (Nguyen et al., 2010). MOPACK is a Java-based optimization framework that supplies a general interface for miscellaneous optimization algorithms. The approach we are presenting uses evolutionary algorithms, which are already implemented in MOPACK. However, a common problem when applying evolutionary algorithms for scheduling problems is the non-consideration of constraints during the generation of child solutions using certain operators. Thus, child solutions often violate given constraints. The paper at hand presents an adjusted operator which enables an improved generation of child solutions. 
Szczesny, Hamm, and König

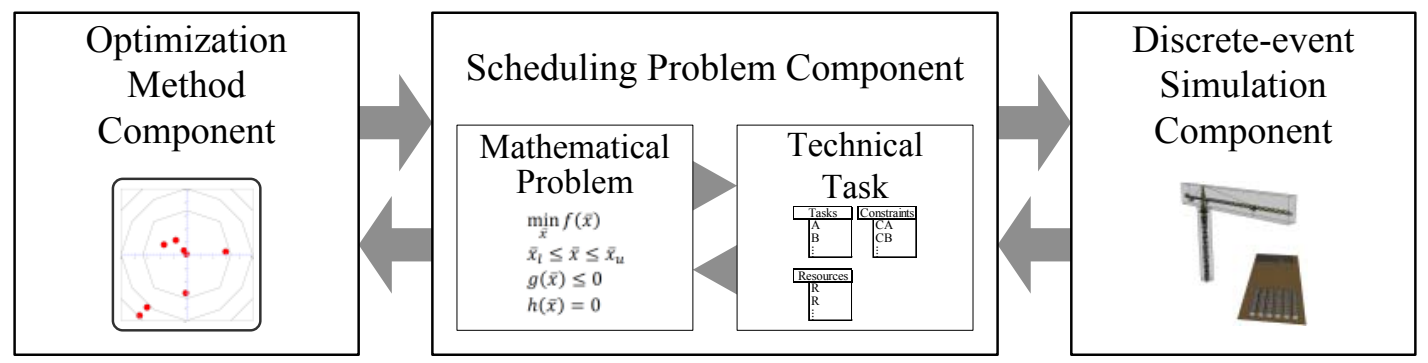

Figure 1: Simulation-based optimization approach for construction scheduling problems.

\section{PROBLEM DESCRIPTION}

The topic of this paper is the generation of efficient schedules for complex construction projects. A valid construction schedule defines the start and finish of every construction activity under consideration regarding existing constraints like precedence relations between activities and resource restrictions. A precedence relation could be the restriction that reinforcement has to be finished before a concreting activity can be started. Resource restrictions define which resources are necessary for the execution of an activity. The resource must be available for the complete execution time of the activity. According to this definition, construction scheduling problems can be generalized as Resource-Constrained Project Scheduling Problems (RCPSP, see Brucker and Knust 2006). An RCPSP consists of a set of activities and a set of renewable resources. Each activity has to be processed without preemption, during the execution a constant amount of specific resources has to be available at all times. Between several activities precedence constraints can be defined, which means that the an activity has to be completed before another activity can be executed. An objective of a RCPSP is to find a schedule with a minimal makespan that meets the constraints imposed by the precedence relations and the limited resource availabilities. Other objectives can be the leveling of the resource utilizations or the optimization of resource idle times. The RCPSP belongs to the class of NP-hard optimization problems.

Since it is not possible to calculate optimal solutions for NP-hard optimization problems, in this paper simulation-based optimization is used for determining efficient schedules. The applied optimization algorithm is an evolutionary algorithm, which implicates that three main steps have to be defined: selection, crossover and mutation. Out of a number of generated solutions in each optimization iteration some good solutions will be chosen in the selection step, the so-called population. Out of these selected solutions, new solutions are generated based on two parent solutions in the crossover step. Considering construction scheduling problems, solutions are represented by valid construction schedules which contain all construction activities. Within the crossover step, two parent solutions, i.e. two valid schedules, are combined to generate a child solution. This crossover process is fundamentally influenced by the crossover operator, which defines how two parent solutions are combined. The number of possible combinations of parent solutions is extremely large. However, using naive crossover operators, most of the combinations of parent solutions will result in invalid child solutions regarding the precedence constraints of the RCPSP. Thus, the solution space, i.e. the set of valid solutions, is very small compared to the number of all combinations. Using a common operator, the probability of generating an unfeasible solution within the crossover process is very large. This problem will be tackled in the paper at hand. An adjusted crossover operator will be presented which improves the generation of child solutions within the crossover step of evolutionary algorithms. Child solutions generated by the so-called adjusted rank-based crossover operator violate given constraints much less frequently. As a result, the probability of generating valid solutions in the crossover step is significantly higher, and the optimization results of the simulation-based optimization process for construction projects are much better. 


\section{METHODOLOGY}

In order to solve the problem described in section three, the following methodology is proposed. The approach couples the concept of constraint-based discrete-event simulation with heuristic optimization methods. In the following subsection, the approach of constraint-based discrete-event simulation is described. Particular attention is paid to the repair mechanism included for invalid solutions. Subsequently, an adjusted crossover operator for optimization methods that is based on evolutionary algorithms is presented and it is described how these two approaches are combined.

\subsection{Constraint-based Discrete-event Simulation}

The application of the constraint-based simulation approach that was introduced to construction scheduling by Beißert, König, and Bargstädt (2007) and König et al., (2007), enables the application of discreteevent simulation for the generation of valid construction schedules that neither violate precedence nor resource constraints. The input parameters for the constraint-based discrete-event simulation are a simulation model as well as the list of construction processes. This list contains a priority-based execution order of the construction processes. The simulation model consists of all construction processes, available resources, material and equipment. These simulation objects are represented by variables. Relations between the variables, e.g. resources and materials that are required for the execution of processes, and execution sequences between processes, are specified by (Beißert, König, and Bargstädt 2009). Based on the simulation input data, the constraint-based discrete-event simulation generates construction schedules that are always valid. This is guaranteed due to the special handling of the input data in form of a prioritybased list of execution orders. This process list is interpreted as a priority list of activities that have to be scheduled. During the simulation, the activity with the highest priority will be executed and required resources are locked. Supposing the activity with the highest priority is not executable due to non-fulfilled precedence or resource constraints, the activity with the next highest priority is checked for its execution ability. This mechanism is shown in Figure 2. Even invalid input data will be repaired and it is guaranteed that generated construction schedules are always valid. Even a randomly generated process order list, i.e., no constraints are considered at all, could be used as simulation input. The construction schedule generated by the constraint-based discrete-event simulation would be a valid schedule. However, there would be nearly no relation between simulation input and output data, i.e., the order of the construction processes would be completely different.

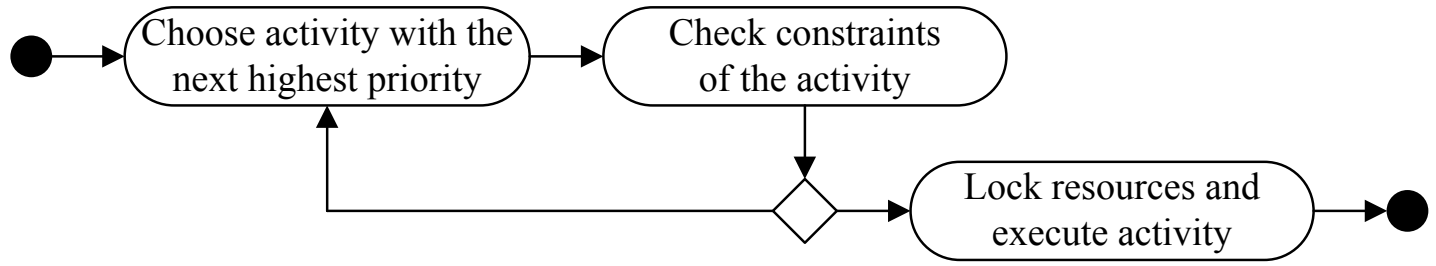

Figure 2: Repair mechanism for invalid solutions within discrete-event simulation

\subsection{Adjusted Crossover Operator for Evolutionary Algorithms}

An optimization method is applied to ensure the generation of near optimal construction schedules. Since the problem class of RCPSP is classified as NP-hard, a calculation of an optimal schedule is not possible. Heuristic optimization methods have proven to be a good choice for finding near optimal solutions. In the proposed concept of simulation-based optimization of construction schedules, an evolutionary algorithm is implemented as a heuristic optimization method.

Because of the general concept of basic evolutionary algorithms, the implemented method has no knowledge about the RCPSP that has to be solved. Thus, for such basic optimization methods, the exploration within the solution space is not efficient. The proposed approach introduces an adjusted and en- 
hanced knowledge-based crossover operator. Using the modified crossover operator, the search within the problem solution space is significantly more efficient and the quality of produced solutions to the RCPSP is greatly improved. The proposed Adjusted Order Crossover-2 operator (AOX2) enhances the Order Crossover-2 (OX2) by Syswerda (1991), which itself is a popular operator for combinatorial optimization problems. The enhancement includes an operator that is able to query knowledge about the specific RCPSP. This specific knowledge consists of the construction processes as well as the precedence constraints of these processes. However, the operator beholds only a special view of this knowledge in form of a rank-based precedence relationship graph (see Figure 3).

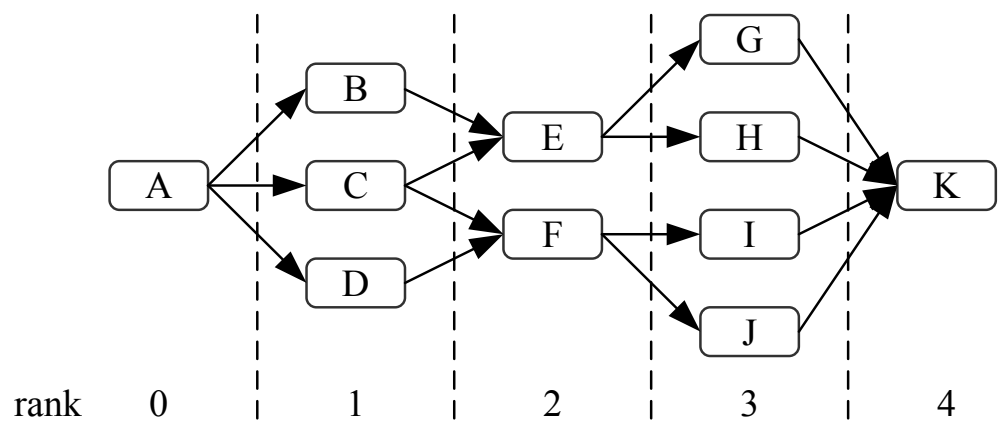

Figure 3: Rank-based precedence relationship graph

Such a rank-based precedence relationship graph $\mathrm{G}$ contains a set of vertices $V=\left\{v_{1}, \ldots, v_{n}\right\}$, where the vertices corresponds to construction processes and a set of edges $E \subseteq\left(V^{2}\right):=\{(x, y) \mid x, y \in V, x \neq$ $y$, where the edges corresponds to relationships between the processes and $(x, y)$ means that vertex $x$ is a predecessor of vertex $y$. Further, this graph is topologically sorted. As a consequence, every vertex with no predecessors is marked with a rank value equal to 0 . Subsequently, every vertex that has only predecessors with a rank value of 0 is marked with a rank value equal to 1 , and so on. The rank-based crossover operator considers the ranks of the activities while recombining parent solutions. As a result, there is a large probability of generating valid solutions as resulting children and there are fewer constraint violations in the resulting children.

First, the encoding that both operators use is explained and then a short description of the OX2 operator with its shortcomings is presented. Thus, how AOX2 functions and the advantages of the AOX2 operator are described as well as the idea behind the rank based precedence constraint graph.

The list of construction processes that have to be executed is presented by a chromosome. The length of each chromosome corresponds to the number of activities that have to be scheduled. Each activity is mapped to an unique integer value that represents one activity. The first integer value entry in each chromosome represents the mapped activity that in turn should be scheduled first if possible; the last chromosome integer value entry represents some other activity that is to be scheduled as the last activity. Thus, the chromosomes' order represents a decreasing priority based representation of integer value mapped activities (see Figure 4).

The OX2 operator is designed to work well with combinatorial optimization problems. The basic idea of OX2 is to preserve the relative order of the chromosome entries within the parents. To achieve this, the first parent chromosome is copied into the child chromosome. Then one-third to one-half of the integer values in the second parent chromosome are selected randomly and their relative order within the second parent chromosome is applied to the child chromosome. Due to the generality of OX2, it is not able to consider problem specific information like precedence constraints. In order to reduce this disadvantage during the position selection, the presented AOX2 operator refers to the knowledge already available in the problem description. Thus, the knowledge of precedence constraints may be provided in form of a topologically sorted graph, the rank based precedence relationship graph. The AOX2 crossover operator 
has the potential to access more detailed optimization problem information such as precedence constraints. Therefore, it considers the rank of each activity instead of selecting the activities randomly. Since activities with equal rank have no precedence constraints among themselves, a permutation of activities with equal rank is considered to be safe in the sense that no precedence constraints are violated. The crossover operator is enhanced to a knowledge-based crossover operator that, in turn, can be used to manipulate the optimization variables in a more sophisticated manner. Thus, the resulting child solution of the crossover step has a greater chance of being a valid solution regarding the precedence relations between construction activities.

solution chromosome
\begin{tabular}{|l|l|l|l|l|l|l|l|}
\hline 4 & 1 & 6 & 3 & 2 & 7 & 5 & 8 \\
\hline 0 & 1 & 2 & 3 & 4 & 5 & 6 & 7 \\
\hline
\end{tabular}
priority

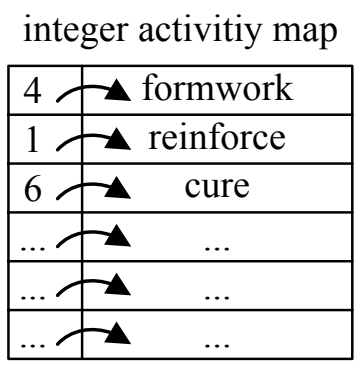

Figure 4: Chromosome encoding

\subsection{Constraint-based Discrete-event Simulation Optimization}

Finally, the previously described methodologies are applied in a simulation-based optimization approach that combines the constraint-based discrete event simulation with a repair mechanism and an evolutionary algorithm optimization method with an adjusted crossover operator. The connection between these two approaches is based on the priority-based list of construction processes. The optimization method is applied to perform a comprehensive search within the problems solution space. During each iteration of the optimization algorithm the preliminary results in form of child chromosomes are transformed into the desired simulation input format. Within the generation of child chromosomes, knowledge about precedence relations is exploited. Then, in turn the discrete event simulation processes this input with regards to precedence, resource as well as material constraints. As a result, the objective function values, i.e., the project duration time, are computed with the side effect that a valid construction schedule is always generated. Hence, one advantage of this approach is a relatively flexible and less complex implementation of an evolutionary optimization algorithm that is coupled with a state-of-the-art constraint-based discrete event simulation. As a result, a convenient search within the problems solution space for near-optimal construction schedules is performed.

\section{IMPLEMENTATION}

In order to implement a number of evolutionary algorithm operators as well as optimization methods, and to be able to compare their results, a software tool is required that allows this task to be fulfilled in a fast, flexible and uncomplicated manner. For this reason, the software framework MOPACK (Multi-method Optimization PACKage) for large-scale simulation-based optimization problems has been designed and developed. MOPACK is a Java-based optimization framework that supplies a general interface for miscellaneous optimization algorithms. In addition, MOPACK provides an API for modeling numerous classes of optimization problems in a declarative and explicit manner using a common interface for optimizers. AOX2 implements the MOPACK operator interface, which provides methods for the modification of a given input, i.e. construction activities sequence. The refinement of OX2 takes place in the integer value selection step. Instead of a random selection of integer value entries in the second parent chromosome, AOX2 randomly selects one integer value entry. The determination of the rank of the selected integer 
value is performed with the help of the rank based precedence constraint graph. Afterwards every activity in the second parent chromosome with equal rank is identified. Then, the relative order of these equal rank selected integer values is applied to the child chromosome. Finally, the rank based selection step is fulfilled until at least one-third of the integer values are permutated. This is carried out to preserve a sensible search within the search domain.

The implementation details are based on the specialized simulation based optimization approach discussed in Hamm et al., (2011). As mentioned in section 4, this implementation makes use of one side effect of the constraint-based discrete-event simulation. Normally, discrete-event simulation computes the objective function value, but as described earlier the side effect that the simulation also computes a valid construction schedule is exploited. Thus, a verification of the performance, i.e. the generated child solutions, of the implemented AOX2 operator is possible.

\section{CASE STUDY AND RESULTS}

In order to prove that the proposed methodology as well as the proposed Adjusted Order Crossover-2 operator solve real world optimization problems efficiently, a comprehensive case study is carried out. The case study consists of the scheduling of shell construction activities of a multi-storey office building.

The aim of the case study is the optimization of the shell construction tasks of two levels of the building with a total of 560 activities, 660 precedence constraints and 552 resource constraints. Table 1 outlines some of the considered activities including their main properties. Figure 5 shows a 3D model top view of the shell construction with column, slab and wall structure elements.

Table 1: Shell construction activities.

\begin{tabular}{lll}
\hline Element type & Activity & Performance factor \\
\hline Column & Install formwork & $0.5 \mathrm{~h} / \mathrm{m}^{2}$ \\
& Reinforce steel & $0.05 \mathrm{~h} / \mathrm{kg}$ \\
& Concreting & $2 \mathrm{~h} / \mathrm{m}^{3}$ \\
& Curing & $8 \mathrm{~h}$ \\
& Removing formwork & $0.3 \mathrm{~h} / \mathrm{m}^{2}$ \\
\hline Wall & Install formwork & $0.3 \mathrm{~h} / \mathrm{m}^{2}$ \\
& Reinforce steel & $0.4 \mathrm{~h} / \mathrm{kg}^{2}$ \\
& Concreting & $0.65 \mathrm{~h} / \mathrm{m}^{3}$ \\
& Curing & $8 \mathrm{~h}$ \\
& Removing formwork & $0.3 \mathrm{~h} / \mathrm{m}^{2}$ \\
\hline Slab & Install formwork & $0.45 \mathrm{~h} / \mathrm{m}^{2}$ \\
& Reinforce steel & $0.4 \mathrm{~h} / \mathrm{kg}^{2}$ \\
& Concreting & $0.5 \mathrm{~h} / \mathrm{m}^{3}$ \\
& Curing & $8 \mathrm{~h}$ \\
& Removing formwork & $0.3 \mathrm{~h} / \mathrm{m}^{2}$ \\
\hline
\end{tabular}

As outlined above, an evolutionary algorithm was implemented using the MOPACK framework. Subsequently, three evolutionary operators had to be implemented. The selection step of the Evolutionary Algorithm was implemented by a Tournament Selection with a tournament size of 5 . For the mutation operator a swap mutation operator was implemented. The mutation probability was set to $1 / 560$. Finally, the operators AOX2 and OX2 were chosen as the crossover operator implementation. For each operator the number of integer values to be interchanged was set to one third of the chromosome length. For the chosen case study this value corresponds to 186 . The population size was set to 50 individuals. During the initialization, each chromosome was computed randomly. The termination criterion consisted of two criterions. The first one is a termination rule that exits the optimization after 500 iterations. The second ter- 
mination criterion is a running mean that exits the optimization when the last 100 iterations show no improvement.

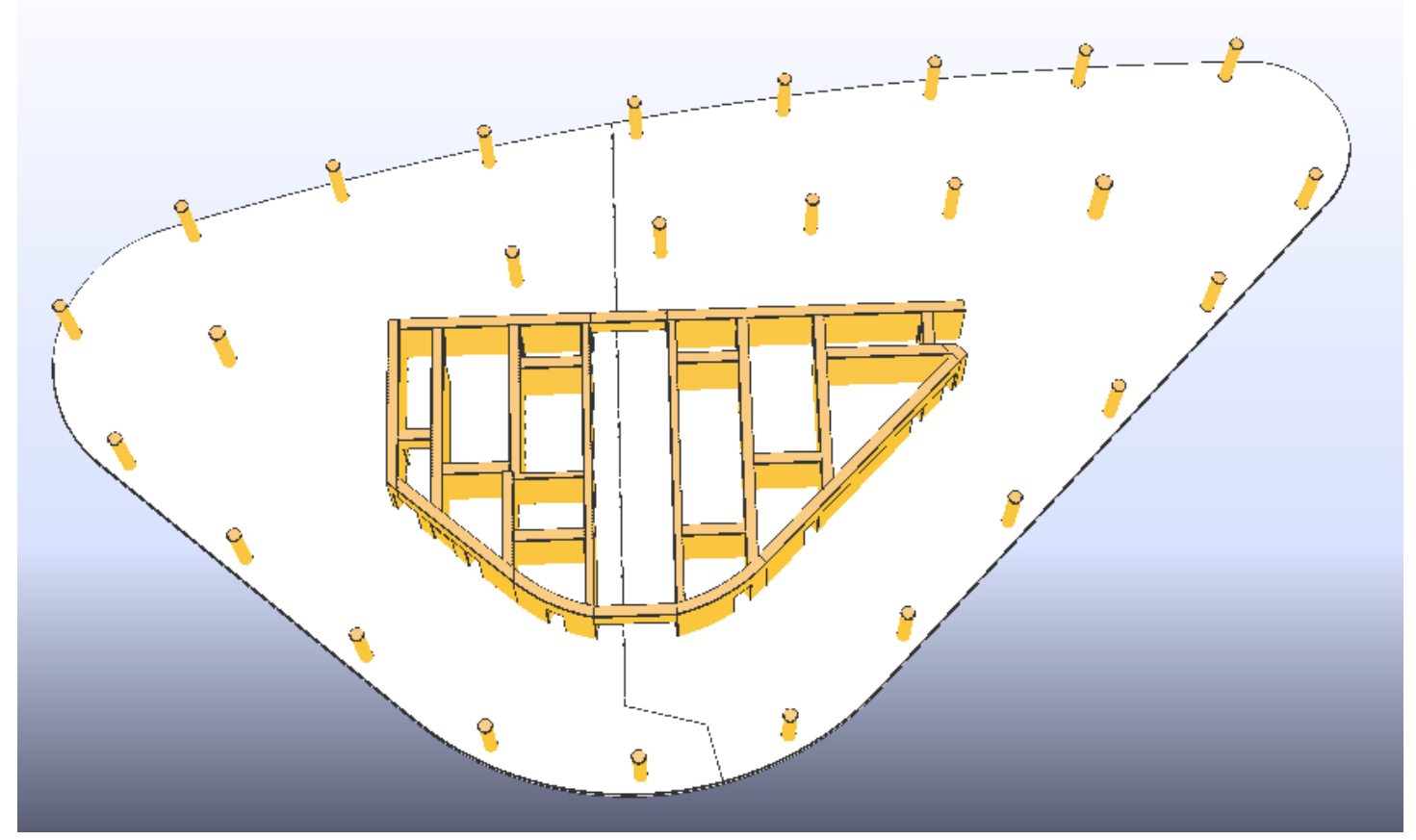

Figure 5: 3D model top view

The optimization results are given in Table 2 and Figure 6. It can be seen that the AOX2 crossover operator outperforms the traditional OX2 crossover operator in two ways. As shown in Table 2, the optimization results computed using the AOX2 crossover operator are better than the results computed using the the OX2 crossover operator. The average fitness value is better. However, more crucial is the value of the experimental standard deviation. The standard deviation using the AOX2 crossover operator is much less than the standard deviation using the OX2. Figure 6 illustrates the optimization progress. The $\mathrm{x}$-axis depicts the numbers of performed iterations while the y-axis indicates the corresponding fitness value. It is obvious, that using the AOX2 crossover operator, a near-optimal solution may be found around 150 iterations earlier than using the OX2 crossover operator. It can be observed that the convergence to a nearoptimal solution is performed earlier and in a much less excursiveness way as using the OX2 operator.

Table 2: Optimization results

\begin{tabular}{|c|c|c|c|c|}
\hline & $\bar{f}$ & $f_{\sigma}$ & $f_{\text {best }}$ & $f_{\text {worst }}$ \\
\hline OX2 & 452.37 & 1.03 & 451 & 454 \\
\hline AOX2 & 450.96 & 0.02 & 450 & 451 \\
\hline
\end{tabular}




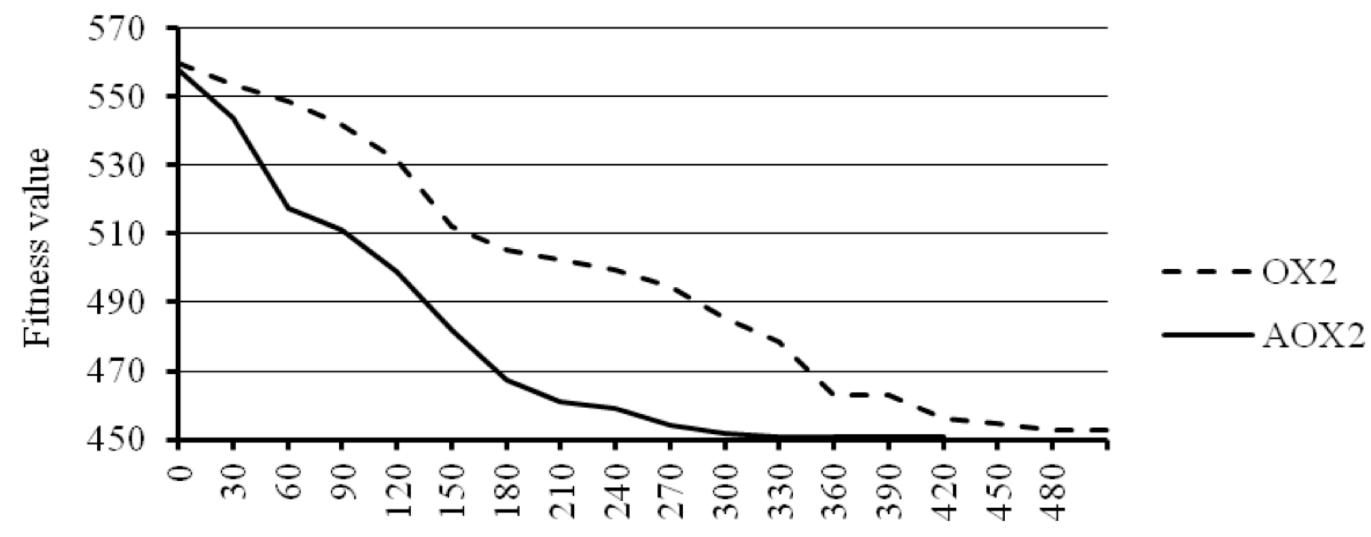

Iterations

Figure 6: Optimization progress

\section{CONCLUSION AND FUTURE WORK}

In this paper, an adjusted rank-based crossover operator for the recombination process for evolutionary algorithms is presented. The algorithm is part of an optimization framework which is used for the generation of efficient schedules for complex construction projects. The Adjusted Order Crossover-2 (AOX2) operator, which is based on the well-known Order Crossover-2 (OX2), is designed to generate child solutions for scheduling problems within the recombination step of evolutionary algorithms. In order to achieve this task effectively, AOX2 makes use of problem specific knowledge and considers precedence constraints between construction activities. These precedence constraints are stored in the rank-based precedence relationship graph. When implementing AOX2, the probability of generating valid child solutions in the recombination step of the evolutionary algorithm is many times greater than the probability when using common operators like the OX2. As a result, the generated schedules are significantly better, i.e. the total duration is significantly shorter, when using the adjusted operator within the optimization framework. A comprehensive case study is presented to obtain comparison results between OX2 and AOX2. It is shown that AOX2 clearly outperforms OX2.

Future research will deal with the comparison of the Adjusted Order Crossover 2 operator with other crossover operators from the literature. A mutation operator will be developed, which will be based on the rank-based precedence relationship graph. It is expected that the optimization results can be improved. The implementation of a more detailed case study will consider outfitting processes. As a result, the variability of construction schedules is much higher which leads to a greater potential for optimization.

\section{REFERENCES}

Bäck, T. 1996. Evolutionary Algorithms in Theory and Practise - Evolutionary Strategies, Evolutionary Programming, Genetic Algorithms. New York: Oxford University Press.

Beißert, U., König, M., and H.-J. Bargstädt. 2007. "Constraint-based simulation of outfitting processes in building engineering." In 24th W78 Conference, Maribor, Slovenia.

Beißert, U., König, M., and H.-J. Bargstädt. 2009. "Considering Quality Aspects for Construction Scheduling using Constraint-Based Simulation." In Proceedings of the 26th International Conference Managing IT in Construction CIB W078, Istanbul, Turkey.

Brucker, P., Drexl, A., Möhring, R., Neumann, K., and E. Pesch. 1999. "Resource-constrained project scheduling: Notation, classification, models, and methods." European Journal of Operational Research 112(1): 3-41.

Brucker, P., and S. Knust. 2006. Complex Scheduling. Berlin: Springer. 
Chen, P.-H., and H. Weng. 2009. "A two-phase GA model for resource-constrained project scheduling." Automation in Construction 18(4): 485-498.

Hamm, M., Szczesny, K., Nguyen, V.V. and M. König. 2011. "Optimization of Construction Schedules with Discrete-Event Simulation using an Optimization Framework." In Proceedings of the 2011 ASCE International Workshop on Computing in Civil Engineering, Edited by Yimin Zhu, and R. R. Issa, 682-689. Reston, Virginia: American Society of Civil Engineers.

Hartmann, S., and R. Kolisch. 2000. "Experimental evaluation of state-of-the-art heuristics for the resource-constrained project scheduling problem." European Journal of Operational Research 127(3): 394-407.

Hartmann, S., and R. Kolisch. 2006. "Experimental investigation of heuristics for resource-constrained project scheduling: An update." European Journal of Operational Research 174(1): 23-37.

Jaśkowski, P., and A. Sobotka. 2006. "Scheduling Construction Projects using Evolutionary Algorithm." Journal of Construction Engineering and Management 132(8): 861-870.

Kennedy, J., Eberhart, R.C., and Y. Shi. 2001. Swarm Intelligence. San Francisco: Morgan Kaufmann Publishers.

Kirkpatrick, S., Gelatt, C.D. and M.P. Vecchi. 1983. "Optimization by Simulated Annealing." Science 220(4598): 671-680.

König, M., Beißert, U., Steinhauer, D., and H.-J. Bargstädt. 2007. "Constraint-based simulation of outfitting processes in shipbuilding and civil engineering." In Proceedings of the 6th EUROSIM Congress on Modelling and Simulation. Wien: ARGESIM - ARGE Simulation News.

Leu, S.-S., and C.-H. Yang. 1999. "A genetic-algorithm-based resource-constrained construction scheduling system." Construction Management and Economics 17(6): 767-776.

Nguyen, V.V., Hartmann, D., Baitsch, M., and M. König. 2010. "A Distributed Agent-based Approach for Robust Optimization." In 2nd International Conference on Engineering Optimization. Lisbon, Portugal.

Senouci, A.B., and N.N. Eldin. 2004. "Use of Genetic Algorithms in Resource Scheduling of Construction Projects" Journal of Construction Engineering and Management 130(6): 869-877.

Syswerda, G. 1991. "Schedule Optimization Using Genetic Algorithms." In Handbook of Genetic Algorithms, Edited by Davis, L., New York: Van Nostrand Reinhold.

Warren Liao, T., Egbelu, P.J., Sarker, B.R., and S.S. Leu. 2011. "Metaheuristics for project and construction management - A state-of-the-art review." Automation in Construction 20(5): 491-505.

\section{AUTHOR BIOGRAPHIES}

KAMIL SZCZESNY is a research associate of Computing in Engineering at Ruhr-Universität Bochum, Germany. He holds a (M. Sc.) degree in Applied Computer Science from the Ruhr-Universität Bochum, German. His research interests include simulation based optimization and evolutionary computation. His email is <kamil.szczesny@rub.de>

MATTHIAS HAMM is a research associate of Computing in Engineering at Ruhr-Universität Bochum, Germany. He received his diploma in Business Mathematics from the Friedrich-Schiller-Universität Jena, Germany in 2008. His research focuses on construction and logistics simulation and simulation based optimization. His email is $<$ matthias.hamm@rub.de>

MARKUS KÖNIG is professor of Computing in Engineering at Ruhr-Universität Bochum, Germany. Previously, he was assistant professor of Theoretical Methods for Project Management at BauhausUniversität Weimar, Germany. He obtained his Ph.D. in Civil Engineering from Leibniz-Universität Hanover, Germany in 2003. His research interests include construction simulation, building information modeling, heuristic optimization techniques, knowledge management in construction, intelligent computing in engineering and computational steering. His email is <koenig@inf.bi.rub.de $>$ 